\title{
Molecular Dynamics Simulations of Collisional Cooling and Ordering of Multiple Charged lons in a Penning Trap
}

J. P. Holder, D. A. Church, L. Gruber, H.E. DeWitt, B. R. Beck, and D. Schneider

This article was submitted to the $16^{\text {th }}$ International Conference on the Application of Accelerators in Research and Industry Denton, TX

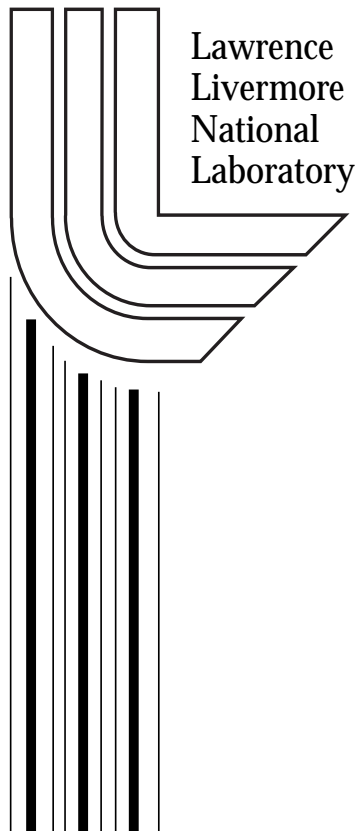

November 1-4, 2000

October 5, 2000 


\section{DISCLAIMER}

This document was prepared as an account of work sponsored by an agency of the United States Government. Neither the United States Government nor the University of California nor any of their employees, makes any warranty, express or implied, or assumes any legal liability or responsibility for the accuracy, completeness, or usefulness of any information, apparatus, product, or process disclosed, or represents that its use would not infringe privately owned rights. Reference herein to any specific commercial product, process, or service by trade name, trademark, manufacturer, or otherwise, does not necessarily constitute or imply its endorsement, recommendation, or favoring by the United States Government or the University of California. The views and opinions of authors expressed herein do not necessarily state or reflect those of the United States Government or the University of California, and shall not be used for advertising or product endorsement purposes.

This is a preprint of a paper intended for publication in a journal or proceedings. Since changes may be made before publication, this preprint is made available with the understanding that it will not be cited or reproduced without the permission of the author.

This report has been reproduced directly from the best available copy.

Available to DOE and DOE contractors from the

Office of Scientific and Technical Information

P.O. Box 62, Oak Ridge, TN 37831

Prices available from (423) 576-8401

http:/ / apollo.osti.gov/bridge/

Available to the public from the National Technical Information Service

U.S. Department of Commerce 5285 Port Royal Rd., Springfield, VA 22161 http://www.ntis.gov/

OR

Lawrence Livermore National Laboratory Technical Information Department's Digital Library http://www.llnl.gov/tid/Library.html 


\title{
Molecular Dynamics Simulations Of Collisional Cooling And Ordering of Multiply Charged Ions In A Penning Trap
}

\author{
J.P. Holder ${ }^{1}$, D. A. Church ${ }^{1}$, L. Gruber ${ }^{2}$, H. E. DeWitt ${ }^{2}$, B. R. Beck ${ }^{2}$, \\ and D. Schneider ${ }^{2}$ \\ ${ }^{1}$ Physics Dept., Texas A\&M University, College Station, TX 77843-4242 \\ ${ }^{2}$ Lawrence Livermore National Laboratory, Livermore CA 94550-0808
}

\begin{abstract}
Molecular dynamics simulations are used to help design new experiments by modeling the cooling of small numbers of trapped multiply charged ions by Coulomb interactions with laser-cooled $\mathrm{Be}^{+}$ions. A Verlet algorithm is used to integrate the equations of motion of two species of point ions interacting in an ideal Penning trap. We use a time step short enough to follow the cyclotron motion of the ions. Axial and radial temperatures for each species are saved periodically. Direct heating and cooling of each species in the simulation can be performed by periodically rescaling velocities. Of interest are Fe ${ }^{11+}$ due to a EUV-optical double resonance for imaging and manipulating the ions, and $\mathrm{Ca}^{14+}$ since a ground state fine structure transition has a convenient wavelength in the tunable laser range.
\end{abstract}

\section{INTRODUCTION}

To study mixed non-neutral plasmas and to prepare for precision fine-structure and hyperfine-structure laser spectroscopy on ground term transitions of highly-charged ions, a beam pulse of multiply charged ions (MCIs) from the electron beam ion trap (EBIT) has been captured into a Penning ion trap (RETRAP) [1] previously filled with cold $\mathrm{Be}^{+}$ions. Typically, these ions have been $\mathrm{Xe}^{44+}$ due to ease of production and the goal of creating strongly coupled cold plasmas, but many other species and charge states can be created and extracted. The $\mathrm{Be}^{+}$ions were similarly captured from a metal vapor vacuum arc (MeVVA) source. These ions were initially cooled to near room temperature by tuning (via trap ring-endcap voltage) their axial oscillation frequency into resonance with a cryogenic parallel tuned circuit. The heating of the parallel circuit by currents induced by fluctuations in the centroid of the confined ion motion, together with ion-ion collisional coupling, initially cools the $\mathrm{Be}^{+}$ ions at an exponential rate with a time constant of several minutes. Eventually, the cooling is dominated by a transverse laser beam de-tuned several $\mathrm{GHz}$ below the cycling resonance transition near $313 \mathrm{~nm}$ [2]. With decreased de-tuning of the cooling laser, the ions then rapidly cooled to below $1 \mathrm{~K}$, as evidenced by a decrease in light scattering, and confirmed by probing the Be ions with a weak second laser tuned through a nearby non-cycling transition.

When the highly charged ions were subsequently captured, electrode switching and collisions with the multiply charged ions initially heated the $\mathrm{Be}^{+}$ions. This heating is associated with an increase in fluorescence rate, which quickly re-cools the $\mathrm{Be}^{+}$ions, and by collisonal coupling, cools the MCIs. As the mixed ion cloud cools, ions with different charge-tomass ratio separate, leaving the cold highly charged $\mathrm{Xe}$ ions in the center of the trap and the $\mathrm{Be}^{+}$ions in an annulus that we image using scattered $313 \mathrm{~nm}$ light. A more detailed account of the procedure and results can be found in ref [3].

It is planned to directly image laser induced fluorescence from a magnetic dipole transition in the ground term of a suitable multiply-charged ion [4]. Of interest are $\mathrm{Fe}^{11+}$ due to a EUV-optical double resonance for imaging and manipulating the ions, and $\mathrm{Ca}^{14+}$ since a ground state fine structure transition ( $\left.2 \mathrm{~s}^{2} 2 \mathrm{p}^{2}{ }^{3} \mathrm{P}_{0}-{ }^{3} \mathrm{P}_{1}\right)$ has a convenient wavelength $(\sim 569$ $\mathrm{nm}$ ) in the tunable laser range. As a guide to future experiments oriented toward direct detection and imaging of the HCI, we have preformed molecular dynamics simulations of multiply-charged ions of potential interest cooled by $\mathrm{Be}^{+}$in a Penning trap. 


\section{SIMULATION}

In preparation, a molecular dynamics code originally developed by E. L. Pollock, and used by $\mathrm{H}$. DeWitt et al. to model strongly coupled plasmas [5] has been modified to exclusively calculate the interaction of two ion species confined in a Penning trap, with separate heating or cooling of each species. The code now runs on personal computers, using the GNU g77 compiler [6].

Simulations of the motion of 5 to 64 multiply charged ions (experimental $\sim 100-500$ ) and 64 to 200 $\mathrm{Be}^{+}$(experimental $\sim 10^{5}$ ) ions were performed using fields, potentials, ion masses, and ion charges correspond to those used in the experimental work. Initially, the ions were arranged in a body-centered cubic lattice, with random assignment of initial locations of each species. The center of mass was placed at the trap center for calculational ease. The ions were assigned pseudo-random initial velocities corresponding to an initial temperature, with the net linear momentum of the ensemble set to zero. The initial ion density, aspect ratio of the lattice, and ion rotation velocity were assigned.

The electrostatic force between each pair of particles was calculated using Coulomb's Law between point charges. The electrostatic trap potential was modeled as an ideal hyperbolic potential, with the voltage applied between ring and end caps of a trap of specified size characterized by a single parameter $\mathrm{q}_{\text {trap }}$. For the $9.34 \mathrm{~mm}$ scale length trap, $\mathrm{q}_{\text {trap }}=-2$ corresponds to about $540 \mathrm{~V}$. This approximates the voltage at which the axial oscillation of a $\mathrm{Be}^{+}$ion is resonant with the $2.5 \mathrm{MHz}$ tuned circuit. The value $\mathrm{q}_{\text {trap }}=-0.039$ corresponds to a shallow axial well, used experimentally for lower ion plasma rotation frequencies, resulting in greater interaction stability between the laser beam and the plasma. Dynamic features associated with ion confinement measurements, such as r.f. excitations, thermal bath coupling to tuned circuits, and image charges, were not modeled.

The equation of motion of the ions was integrated in the lab frame using the Verlet method [7], based on the Stoermer formula $\mathbf{r}_{\mathrm{i}}(\mathrm{t}+\tau)=2 \mathbf{r}_{\mathrm{i}}(\mathrm{t})-$ $\mathbf{r}_{\mathrm{i}}(\mathrm{t}-\tau)+\tau^{2} \mathbf{a}_{\mathrm{i}}(\mathrm{t})+0\left(\tau^{4}\right)$. Here $\mathbf{a}_{\mathrm{i}}$ is the acceleration of ion $\mathrm{i}$ due to the electric and magnetic forces of the trap, as well as the Coulomb forces. Since the trap symmetry axis is aligned with the uniform magnetic field $\mathrm{B}$, this equation is simplest to apply in the $\mathrm{z}$ coordinate. For the $\mathrm{x}$ and $\mathrm{y}$ radial coordinate, the time-centered velocity $\mathbf{v}_{\mathrm{i}}(\mathrm{t})=\left(\mathbf{r}_{\mathrm{i}}(\mathrm{t}+\tau)-\mathbf{r}_{\mathrm{i}}(\mathrm{t}-\tau)\right) / 2 \tau+$ $O\left(\tau^{3}\right)$ was used to evaluate the Lorentz force in the Stoermer formula and to get implicit expressions for $\mathrm{x}(\mathrm{t}+\tau)$ and $\mathrm{y}(\mathrm{t}+\tau)$ in terms of present acceleration, $\mathrm{x}$ and $y$ position, and a single past time step of stored past $\mathrm{x}$ and $\mathrm{y}$ positions. The parameter $\tau$ was chosen to be a fraction of the period of the initial plasma oscillation, so that it is also small enough to follow the largest cyclotron frequency of the simulation. It was found that small values of $\tau(\sim 30-300 \mathrm{ps}$ compared to $\sim 150 \mathrm{~ns} \mathrm{Be}^{+}$cyclotron period) resulted in a stable simulation start.

The mean squared axial velocity component (proportional to axial temperature $\mathrm{T}_{\mathrm{z}}$ of each species) is averaged for every other step. Every 1000 steps, an estimate of the rigid body rotation frequency was made, and in that reference frame, the mean squared radial velocity for each species (proportional to $T_{r}$ ) was calculated and recorded, along with the fixed frame kinetic energies and potential energies of the ions. When simulating cooling, if the running average axial temperature of an ion species exceeds the specified temperature by $3 \%$, the former z-axis positions of the species being "cooled" (proportional to axial velocity in a harmonic well) were re-scaled using a Taylor series expansion to approach the desired temperature. The other ion species could also be "heated" axially by a similar scaling, allowing a fixed amount of kinetic energy to be added on average to each ion. The temperature of the heated species can approach a steady state with the cooled species. Typically, the target temperature is lowered in steps as the system approaches quasi-steady state.

\section{RESULTS}

Major results of the simulation include observation of the onset of properties of a highly magnetized nonneutral plasma [8,9]. As ions are cooled in a magnetic field, the magnetic moment of the cyclotron motion eventually becomes an adiabatic invariant. This invariance occurs because the amplitudes of the Fourier components of a typical Coulomb collision become too low to excite the cyclotron motion. The invariance is broken in close, high velocity collisions. The multiply charged ions, with higher cyclotron frequencies, exhibited this effect at higher temperatures than the $\mathrm{Be}^{+}$ions, ( ranging from $40 \mathrm{~K}$ to $2 \mathrm{~K}$ for $\mathrm{Xe}^{44+}$ ) depending on density (or equivalently cloud rotation frequency), as the "radial cyclotron temperature" decoupled at an exponential rate from the "axial temperature". For example, a simulation of 186 
$\mathrm{Be}^{+}$ions and $64 \mathrm{Fe}^{11+}$ ion in a $\mathrm{q}_{\text {trap }}=-2.0$ well and with a cold rigid body rotation frequency of $592 \mathrm{kHz}$, this temperature was about 2 Kelvin. A snapshot of this simulation is shown in Figures 1 and 2. The ions are separated, as discussed below. However, the radial and axial motions remain coupled through the ion cloud rotation. The $\mathrm{Be}^{+}$ions were not significantly magnetized for simulated temperatures $>1$ Kelvin.

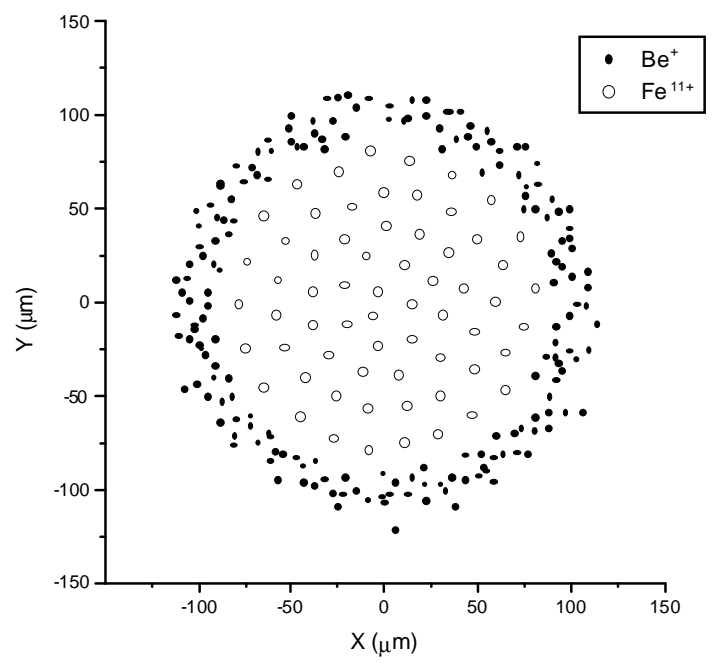

FIGURE 1. A "snapshot" $x-y$ projection of the cloud of $186 \mathrm{Be}^{+}$and $64 \mathrm{Fe}^{11+}$ ions. The trap parameter is $\mathrm{q}$ $=-2.0$ corresponding to about 540 Volts applied to our trap. The $\mathrm{Be}^{+}$ions are axially cooled to 1 Kelvin. The rotation frequency of the cloud is approximately 592 $\mathrm{kHz}$

Typically, the multiply charged ions and $\mathrm{Be}^{+}$ions centrifugally separate, as Figures 1 and 2 show. This centrifugal separation occurs when the plasma rotates as a rigid body. The "pseudopotentials" associated with the plasma rotation for each species then differ, because of the different mass-to-charge ratios, causing the ions with higher mass to charge ratio (in this case $\left.\mathrm{Be}^{+}\right)$to move radially outward and separate from the MCIs. The separation temperature was found to depend on the trap parameter $\mathrm{q}_{\text {trap }}$ and rotation frequency (bounds of which depend on $\mathrm{q}_{\text {trap }}$ ) as expected, although the current code (no spacial averaging / small numbers of particles) and mode of simulation (step down of set temperature) does not track the separation in detail.

A particular case of $64 \mathrm{Al}^{3+}$ ions and $64 \mathrm{Be}^{+}$ions, for which the mass to charge ratios of each species are identical, was simulated in a $\mathrm{q}_{\mathrm{trap}}=-0.5$ trap. Here at milliKevin temperatures a charge separation is observed [9]. This is shown in Figure 3. Simultaneously ordering of the MCIs can be seen, first as shells, then, at lower temperatures, rings of ions are apparent. The shell surfaces then have the characteristic triangular structure of 2D Coulomb systems [7,10].

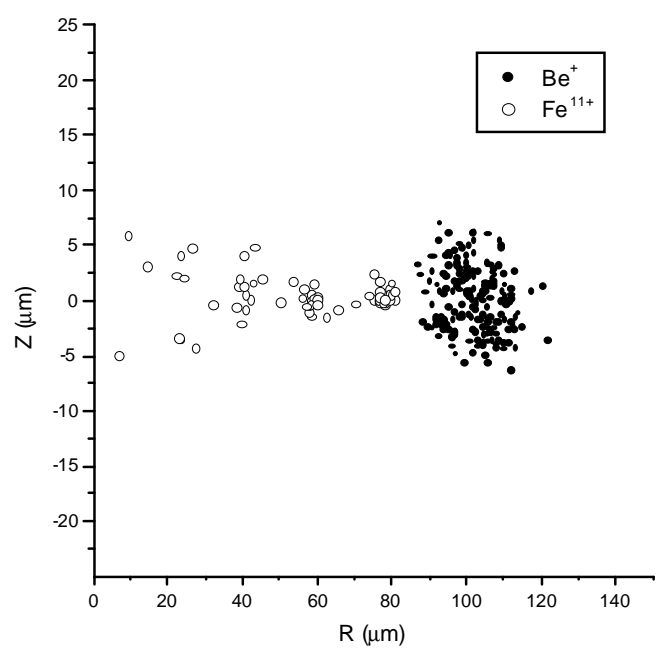

FIGURE 2. A “snapshot” cylindrical R-z projection of the ion cloud described in Figure 1.

Additional work involves the study of the effect of heating sources on the multiply charged ion temperatures given an experimentally measurable $\mathrm{Be}^{+}$ temperature. From simulated motion and atomic data, spectroscopic lineshapes can be calculated and parameters for future experiments estimated. For example, $\mathrm{Ca}^{14+}$ has been caught and electronically cooled in our trap. Unfortunately, $\mathrm{Be}^{+}$laser cooling was not functioning at that time due to laser failure. Calculations are proceeding to model this system to optimize our procedure for a future experimental opportunity.

Further work is suggested by Figure 4, where the motion of the ordered ions might be used to quantum entangle the fine structure states of a few multiply charged ions using an appropriate laser. An ion state then could be read out using a EUV or x-ray electric dipole transition. 


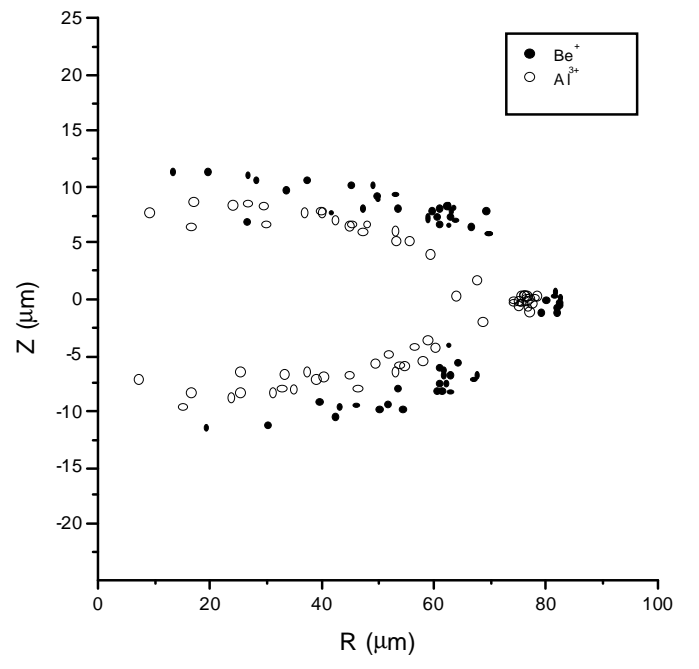

FIGURE 3. A "snapshot" cylindrical R-z projection of the ion cloud of $64 \mathrm{Be}^{+}$and $64 \mathrm{Al}^{3+}$ ions. The axial temperatures are $\sim 2.5$ milliKelvin while the rotational temperatures are $\sim 640$ milliKelvin. Trap parameter is $\mathrm{q}=-0.5$ and ion cloud rotation frequency is $\sim 163 \mathrm{kHz}$.

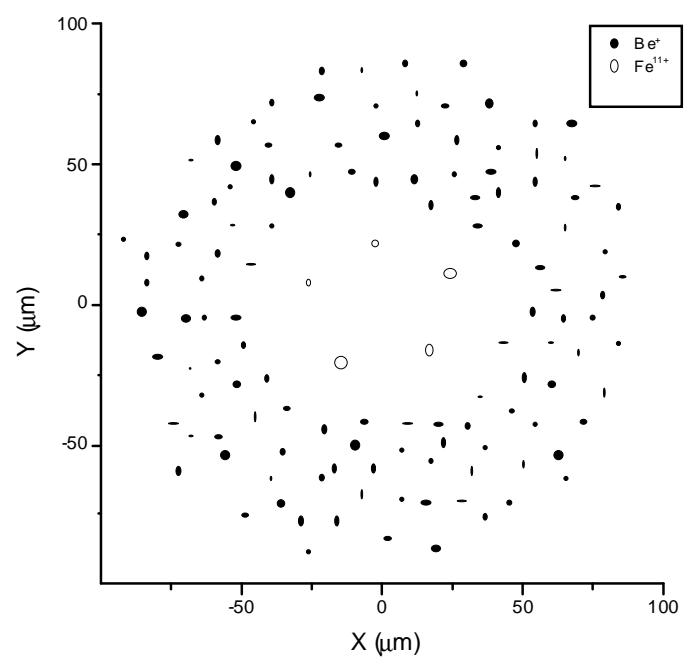

FIGURE 4. A "snapshot" $x-y$ projection of the cloud of $123 \mathrm{Be}^{+}$and $5 \mathrm{Fe}^{11+}$ ions. The trap parameter is $\mathrm{q}=-$ 2.0 corresponding to about 540 Volts applied to our trap. The $\mathrm{Be}^{+}$ions are axially cooled to 0.25 Kelvin. The rotation frequency of the cloud is approximately $544 \mathrm{kHz}$

\section{ACKNOWLEDGMENTS}

This work was Supported through NSF grant PHY9876899, and State of Texas Advanced Research Program \#010366-0018-1997.

Work was performed under the auspices of the U.S. Department of Energy by University of California Lawrence Livermore National Laboratory under contract No. W-7405-ENG-48 and supported in part by the U.S. Department of Energy, Basic Energy Sciences (Chemical Sciences).

\section{REFERENCES}

1. Schneider, D. et al, Rev. Sci. Instrum. 65, 3472 (1994).

2. Brewer, L.R. et al, Phys. Rev. A 57, 859 (1988).

3. Gruber, L. et al, submitted to $P R L$

4. Church, D. A. et al, "RETRAP: An Ion Trap for Laser Spectroscopy of Highly-Charged Ions" in Trapped Charged Particles and Fundamental Physics, edited by D.H.E. Dubin and D. Schneider, AIP Conference Proceedings 457, New York: American Institute of Physics, 1999, pp. 235-241.

5. DeWitt, H.E., Slatterly, W.L. and Yang, J., "Monte Carlo Simulation of the OCP Freezing Transition," in Strongly Coupled Plasma Physics, edited by H. M. Van Horn and S. Ichimaru, Rochester: University of Rochester Press, 1993, pp. 425.

6. www.gnu.org

7. Verlet, L., Phys. Rev. 159, 98 (1967).

8. Peurrung, A.J., Kouzes, R.T. and Barlow, S.E., Int. J. Mass Spectrom. Ion Processes 157/158, 39 (1996).

9. Dubin, D. H. E. and O’Neil, T. M., RMP 71, 87 (1999).

10. Hasse, R.W. and Schiffer, J.P., Ann. of Phys. 203, 419 (1990). 